\title{
SIMULATION OF X-RAY POWDER DIFFRACTION PATTERNS USING DYNAMICAL THEORY
}

\author{
Muniz, F.T.L. ${ }^{1 *}$, Lima, A.N.C. ${ }^{2}$, Batista, A.M.L. ${ }^{2}$, Sasaki, J.M. ${ }^{1}$ \\ ${ }^{1}$. Department of physis, Federal University of Ceará, Fortaleza, Ceará, Brasil \\ *tiagomunizsq@gmail.com \\ 2. Department of Metallurgical and Materials Engineering, Federal University of Ceará, Fortaleza, Ceará, Brasil
}

\begin{abstract}
The dynamical theory of X-ray diffraction is a theory that unlike the kinematic theory has its origin in Maxwell's equations and Bragg's law. This theory has more complete and adequate physical treatment taking into account all interactions between electromagnetic wave inside the crystal and hence also the physical properties of the medium[1]. These interactions bring effects that are neglected in the kinematic theory, which is more traditional and most commonly used when dealing with thin crystal[2]. In this work has written a program in order to simulate the diffraction profiles peaks using the equation of reflectivity, this which relates the diffracted and incident waves provided by the dynamical theory, to calculate the peak profile. The diffraction profiles were calculated based on a distribution of crystal sizes represented by a lognormal function. Afterwards, was made the convolution this function, said structural function with another function which, in turn, represents the convolution of all instrumental functions, thereby obtaining the resultant profiling shown in this work[3]. The calculations were applied on samples of silicon, $\mathrm{CeO}_{2}$ and $\mathrm{LaB}_{6}$.
\end{abstract}

[1] Authier, A. Dynamical Theory of X-Ray Diffraction. Oxford Science Publications, IUCr, (2001).

[2] Zachariasen, W., Theory of X-Ray Diffraction in Crystals. Dover Publications, Inc., New York (1945).

[3] R. W. Cheary, A. A. Coelho, Fundamental Parameters Line Profile Fitting in Laboratory Diffractometers, Journal of Research of the National Institute of Standards and Technology, 109, 1, (1- 25) 2004. 